\title{
Synnemata Production by Isaria tenuipes using Colored Cocoon Silk- worm, Golden Silk
}

\author{
In-Pyo Hong*, Pil-Don Kang, Kee-Young Kim, Man-Young Lee, Yong-Soo Choi, Nam-Suk Kim, Hye-Kyung \\ Kim, Sung-Hee Nam, and Kwang-Gill Lee \\ Sericultural \& Apicultural Materials Division, National Academy of Agricultural Science (NAAS), Suwon 441-100, Korea
}

(Received 22 October 2010; Accepted 06 December 2010)

This study was conducted to test the use of colored cocoon silkworm, Golden Silk, as a host for the fruiting body production of Cordyceps mushroom. Golden Silk variety had a shorter larval period than Yangwonjam at $25.3^{\circ} \mathrm{C}$ and $78.5 \% \mathrm{RH}$ : The average larval period of the Golden Silk was 24 days and $7 \mathrm{hr}$, and that of the Yangwonjam was 24 days and $15 \mathrm{hr}$. In the infection test of $I$. tenuipes on silkworm, the larval period of normal silkworms was longer than the silkworms inoculated with $I$. tenuipes in both Golden Silk and Yangwonjam. The pupae survival percentage of Golden Silk was 94.4\%, whereas it was 91.9\% in Yangwonjam. Golden Silk had a higher pupation rate than Yangwonjam. The pupation rate of normal silkworms was about 5\% higher than that of silkworms inoculated with $I$. tenuipes. Infection rate of $I$. tenuipes into larvae of 5 th instar newly exuviated silkworm was $93.8 \%$ in Golden Silk, and $96.9 \%$ in Yangwonjam. Synnemata production of $\boldsymbol{I}$. tenuipes was better in Yangwonjam with an incidence rate of $99.8 \%$ than Golden Silk with $\mathbf{9 8 . 6} \%$. The average synnemata weight of Golden Silk was $1.12 \mathrm{~g}$ and that of Yangwonjam $1.29 \mathrm{~g}$. Golden Silk had a lower synnemata weight than Yangwonjam. But no significant difference was observed in synnemata weight varieties. The synnemata of $I$. tenuipes produced on pupae were white or milky-white in color, and similar in shape and color to wild synnemata collected in Korea.

Key words: Bombyx mori, Golden Silk, Isaria tenuipes, Spraying, Synnemata

\footnotetext{
*To whom the correspondence addressed

Sericultural \& Apicultural Materials Division, National Academy of Agricultural Science (NAAS), Seodun-dong, Gwonseon-gu, Suwon-si, Gyeonggi-do, 441-100, Korea

Tel: +82-31-290-8461; Fax: +82-31-290-8503;

E-mail: iphong20@korea.kr
}

\section{Introduction}

Sericulture was one of the most important cottage industries in Korea in 1990's. Many researchers have tried to breed the varieties of silkworm (Bombyx mori L.) to improve the quantity and quality of raw silk in 1990s. But after the declination of sericulture industry, sericulture farmers have had an attention on the health foods or dietary supplement such as silk powder and male pupa. These demands have forced the development of the new silkworm varieties suitable for special purposes. Recently new silkworm varieties have been bred for these purposes in Korea: Yangwonjam with a sex-limited larval marking for mass production of male pupae (Kang et al, 2000), Hachojam with non-cocooning silkworm variety for synnemata production of Cordyceps mushrooms (Kang et al., 2002), Golden Silk making yellow color cocoon (Kang et al., 2007), and Eolrukmal and Hukpyobeom with peculiar marks in larval stage for an education and experience (Kang et al., 2010a) were bred, respectively.

Biological and pharmaceutical compounds such as cordycepin and $\mathrm{N}^{6}$-(2-hydroxyethyl) adenosine were reported in Cordyceps mushroom (Furuya et al., 1983; Hsu et al., 2002; Li et al., 2003). Artificial culture techniques have been first developed for the fruiting body production of Cordyceps mushroom (Dongchunghacho) using silkworm as a host in Korea (Ha et al., 2005). Many researchers have tried to find silkworm varieties suitable for the fruiting body production of these fungi. The Golden Silk was bred for a deluxe clothes, shroud, wallpaper, and handicraft. Handicraftsmen have used cocoons only as handicraft materials except pupae. Therefore we tested the larval period and pupation rate of Golden Silk, the infection rate and incidence rate of Isaria tenuipes at 8 local areas, and finally confirmed the possibility use of Golden Silk as a host for the fruiting body production of Cordyceps mushroom. 

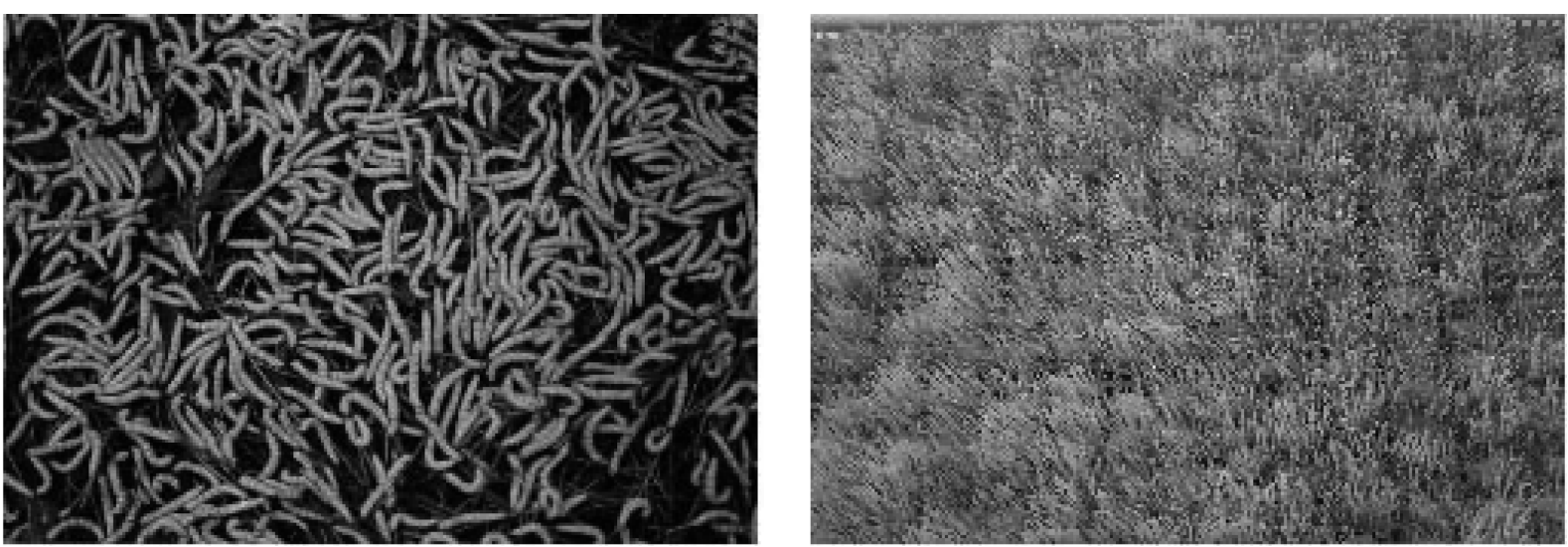

Fig. 1. Larvae of 5th instar newly exuviated silkworm (A), and synnemata formed on Golden Silk pupae (B).

\section{Materials and Methods}

\section{Host insect}

Two kinds of silkworm varieties of B. mori, Golden Silk and Yangwonjam as control group, were used for synnema formation by $I$. tenuipes. Golden Silk is an F1 hybrid between the Japanese race Jam 311 and the Chinese race Jam 312 in 2006 (Kang et al., 2007). The Japanese race pure line Jam 311 (breeding line JS 169) was crossed between stock lines of Jam125, and Kyunsakjuk and Huka in 2002, and the Chinese pure line Jam 312 (breeding line CS 186) was crossed between stock lines of Jam 304 and Goechuk in 2003. Yangwonjam is an F1 hybrid between the Japanese race Jam 144 and the Chinese race Jam in 1999 (Kang et al., 2000). The Japanese race pure line Jam 144 (breeding line JS 129) was crossed between stock lines of P 8503 and H5R1 in 1993, and the Chinese pure line Jam 144 (breeding line CS 98) was crossed between stock lines of M 8312 and 8190 in 1984. The crossed silkworms were reared with natural mulberry leaves by the guide book of the silkworm rearing of the National Academy of Agricultural Science, Rural Development Administration (RDA), Korea.

\section{Fungal strain}

Strain of I. tenuipes used in this study was isolated from conidiospores of a specimen collected at Mt. Halla on Jeju Island in July 2003.

\section{Inoculum preparation}

The conidiospores of I. tenuipes were obtained from unconverted rice grain medium. The medium was made by mixing $150 \mathrm{~g}$ of unconverted rice grain, $15 \mathrm{~g}$ of silkworm pupae powder, and $80 \sim 100 \mathrm{~mL}$ of distilled water. The mixture was poured into a $500 \mathrm{~mL}$ flask, and each flask was sterilized at $121^{\circ} \mathrm{C}$ for $20 \mathrm{~min}$ and inoculated with mycelial discs $(5 \mathrm{~mm})$ of 1 . tenuipes from the growing margins on potato dextrose agar medium. The inoculated media were cultured at $25^{\circ} \mathrm{C}$ for 30 days and shaken at three-day intervals to produce mass conidia (Ha et al., 2005). The conidiospores formed on unconverted rice grain media were harvested using a sterile inoculation loop and added with $0.2 \mathrm{~mL}$ of $0.02 \%$ Tween 20 solution to uniformly disperse the spore suspension.

\section{Inoculation}

The concentration of the spore suspension was adjusted with sterilized distilled water to $10^{8}$ spores/ $\mathrm{mL}$, and the number of spores was counted with a hematocytometer (Superior, Marienfeld, Germany). The spore suspension added with starch syrup for an easy attachment on the silkworm was inoculated on each larvae of 5 th instar newly exuviated silkworm using the spraying method (Fig. 1 A). Each test was sprayed three times at 12-hr intervals (Ha et al., 2005). The inoculated silkworms were reared with natural mulberry leaves until mounting of the silkworm, as directed by the silkworm rearing guidebook of the National Academy of Agricultural Science, RDA, Korea.

\section{Induction of endosclerotium and synnemata}

The cocoons were harvested at 11 days after mounting from the cocoon frames. The silkworm pupae were picked out from the cocoons after cutting the tops with a knife the following day. The infected pupae were placed on a wet cotton cloth at $1-\mathrm{cm}$ square distance in transparent plastic containers. Each container with the inoculated pupae was kept in a growth room controlled at $20 \sim 22^{\circ} \mathrm{C}$ and a relative humidity $(\mathrm{RH})$ of about $95 \%$ under a dark condition to induce synnemata formation. The containers were supplied with water periodically to prevent excessive drying. 
Table 1. Larval period of the silkworm varieties bred

\begin{tabular}{ccc}
\hline Hybrid & $25.3^{\circ} \mathrm{C}$ and $78.5 \%$ relative humidity \\
\hline Hybrid & \multicolumn{2}{c}{ Larval period (days. hr) } \\
\hline Hybrid & Normal silkworm & $\begin{array}{c}\text { Inoculated } \\
\text { silkworm }\end{array}$ \\
\hline Golden Silk & $24.07 \pm 0.16$ & $24.03 \pm 0.18$ \\
\hline Yangwonjam & $24.15 \pm 0.65$ & $24.07 \pm 0.16$ \\
\hline
\end{tabular}

Table 2. Pupation rate by silkworm variety

\begin{tabular}{ccc}
\hline Hybrid & \multicolumn{2}{c}{ Pupation rate (\%) } \\
\hline Hybrid & Normal silkworm & $\begin{array}{c}\text { Inoculated } \\
\text { silkworm }\end{array}$ \\
\hline Golden Silk & $94.4 \pm 5.73$ & $89.4 \pm 7.24$ \\
\hline Yangwonjam & $91.9 \pm 8.54$ & $84.7 \pm 9.07$ \\
\hline
\end{tabular}

\section{Results and Discussion}

\section{Larval period}

The average larval period of the Golden Silk was 24 days and $7 \mathrm{hr}$, and Yangwonjam was 24 days and $15 \mathrm{hr}$ at $25.3^{\circ} \mathrm{C}$ and $78.5 \%$ RH (Table 1). Golden Silk variety had a shorter larval period than Yangwonjam. In the infection test of I. tenuipes on silkworm, the Golden Silk sprayed with $I$. tenuipes had 24 days and $3 \mathrm{hr}$, and the Yangwonjam 24 days and $7 \mathrm{hr}$ in the average larval period. The larval period of normal silkworms was longer than the silkworms inoculated with I. tenuipes in both Golden Silk and Yangwonjam. It was assumed that the developmental period was shortened because of the physiological changes in the silkworm from the fungi inoculation (Yun, 2005). Our results agree that Hachojam inoculated with $I$. tenuipes had a shorter larval period than normal silkworms (Kang et al., 2010b), though using different silkworm variety.

\section{Pupation rate}

The pupae survival percentage of Golden Silk was 94.4\%, whereas it was 91.9\% in Yangwonjam (Table 2). Golden Silk had a higher pupation rate than Yangwonjam. Silkworms spin cocoons around themselves and become pupae within the cocoon. But some of the silkworms died within the cocoon or missed from the cocoon frame during the rearing period due to various reasons. The pupation rate was calculated with the number of healthy pupae within cocoons that were made after mounting of the mature larvae. The pupation rate of normal silkworms was about 5\% higher than that of silkworms inoculated with $I$. tenuipes. In silkworms sprayed with I. tenuipes, 1786 normal pupae were harvested from 1999 silkworms in Golden Silk (89.4\%), and 1693 silkworms from 2000 in
Table 3. Infection rate of Isaria tenuipes by silkworm variety

\begin{tabular}{cccc}
\hline Hybrid & $\begin{array}{c}\text { No. of silkworm } \\
\text { tested }\end{array}$ & $\begin{array}{c}\text { No. of pupae } \\
\text { infected }\end{array}$ & $\begin{array}{c}\text { Infection } \\
\text { rate (\%) }\end{array}$ \\
\hline Golden Silk & 1803 & 1691 & $93.8 \pm 2.7$ \\
\hline Yangwonjam & 1726 & 1672 & $96.9 \pm 3.1$ \\
\hline
\end{tabular}

Table 4. Synnemata formation of Isaria tenuipes by silkworm variety

\begin{tabular}{cccc}
\hline Hybrid & $\begin{array}{c}\text { No. of pupae } \\
\text { tested }\end{array}$ & $\begin{array}{c}\text { No. of pupae } \\
\text { produced }\end{array}$ & $\begin{array}{c}\text { Incidence rate } \\
(\%)\end{array}$ \\
\hline Golden Silk & 1687 & 1663 & $98.6 \pm 2.0$ \\
\hline Yangwonjam & 1669 & 1665 & $99.8 \pm 0.3$ \\
\hline
\end{tabular}

Yangwonjam (84.7\%). Golden Silk had a higher pupation rate than Yangwonjam.

\section{Infection}

After cutting the harvested cocoons, the pupae infected with I. tenuipes were counted by hardness. Infection rate of I. tenuipes into larvae of 5th instar newly exuviated silkworm was good in both of Golden Silk and Yangwonjam at following three sprays (Table 3). Golden Silk was $93.8 \%$, and Yangwonjam 96.9\% in an Infection rate.

\section{Synnema formation}

Synnemata were induced from infected pupae in the growth room with a temperature of $18 \sim 20^{\circ} \mathrm{C}$ and $\mathrm{RH}$ of about $95 \%$ under a dark condition. The synnemata of $I$. tenuipes were produced from almost all pupae tested (Fig. 1 B). Synnema production of I. tenuipes was better in Yangwonjam with an incidence rate of $99.8 \%$ than Golden Silk with $98.6 \%$ (Table 4). But no significant difference was observed in synnemata formation among the silkworm varieties.

\section{Synnemata characteristics}

The average synnema weight of Golden Silk was $1.12 \mathrm{~g}$, and Yangwonjam was 1.29 g. Golden Silk had a lower synnemata weight than Yangwonjam. But no significant difference was observed in synnemata weight among the silkworm varieties. The number of synnemata with over 3 $\mathrm{cm}$ long ranged from 13 to 16 (mean $=13.3$ ) on a pupa. Twelve synnemata were produced from a pupa of Golden Silk, and 14 from Yangwonjam. The colour of these Synnemata produced in this experiment was white or milky white (Fig. 1B). The synnemata of I. tenuipes produced on pupae were similar in shape and color to wild synnemata collected in Korea (Sung, 1996).

In conclusion, these findings suggest that the pupae of Golden Silk can be used as a host for the fruiting body 
Table 5. Characteristics of synnemata by silkworm variety

\begin{tabular}{ccc}
\hline Hybrid & Synnema weight $(\mathrm{g})$ & No. of synnemata \\
\hline Golden Silk & $1.12 \pm 0.3$ & $12 \pm 3.9$ \\
\hline Yangwonjam & $1.29 \pm 0.3$ & $14 \pm 5.9$ \\
\hline
\end{tabular}

production of Cordyceps mushroom, and also its cocoon as handicraft materials.

\section{Acknowledgements}

This research was supported by a grant from the Korean Rural Development Administration (Agenda Program, PJ0067792010).

\section{References}

Furuya T, Hirotani M, Matsuzawa M (1983) N6-(2-hydroxyethyl) adenosine, a biologically active compound from cultured mycelia of Cordyceps and Isaria species. Phytochemistry 22, 2509-2512.

Ha NG, Kim SY, Kang JH, Kang PD, Sung GB and Hong IP (2005) Biological activities and cultural characteristics of an Entomogenous fungus, Paecilomyces tenuipes (Peck) Samson. Korean J Seric Sci. 47, 12-17.

Hsu TH, Shiao LH, Hsieh CH and Chang DM (2002) A comparison of the chemical composition and bioactive ingredients of the Chinese medicinal mushroom DongChongXiaCao, its counterfeit and mimic, and fermented mycelium of Cordyceps sienesis. Food Chemistry 78, 463-469.

Kang PD, Kim KM, Sohn BH, Woo SO, Ryu KS (2000) Breeding of Yangwonjam, a both parent sex-limited larval marking variety suitable for spring and autumn rearing season. Korean J. Seric. Sci. 42, 24-27.

Kang PD, Sohn BH, Lee SU, Hong SJ (2002) Breeding of a new non-cocooning silkworm variety, Hachojam, suitable for autumn rearing season. Int. J. Indust. Entomol. 4, 77-81.

Kang PD, Lee SU, Jung IY, Sohn BH, Kim YS, Kim KY, Kim MJ, Hong IP, Lee KG, Park KY (2007) Breeding of new silkworm Variety Golden Silk, a Yellow cocoon color for spring rearing season. Korean J. Seric. Sci. 49, 14-17.

Kang PD, Jung IY, Kim KY, Kim MJ, Sohn BH, Lee KG (2010a) Breeding of two new silkworm Varieties with peculiar larval mark, Eolrukmal and Hukpyobeom. Int. J. Indust. Entomol. 20, 115-116.

Kang PD, Sung GB, Kim KY, Kim MJ, Hong IP, Ha NG (2010b) Breeding of a silkworm Variety for synnemata production of Isaria tenuipes. Kor. J Mycol. 38, 180-183.

Li SP, Zhao KJ, Ji ZN, Song ZH, Dong TTX, Lo CK, Cheung JKH, Zhu SQ, Tsim KWK (2003) A polysaccharide isolated from Cordyceps sinensis, a traditional Chinese medicine, protects PC12 cells against hydrogen peroxide-induced injury. Life Science 73, 2503-1513.

Sung JM (1996) The Insects-born fungus of Korea in color, pp. 62-72, Kyohak Publishing Co Ltd., Seoul.

Yun JS (2005) Effect of Cordyceps, Paecilomyces sp., Cordyceps pruinosa and Paecilomyces japonica, on the development of domestic silkworm, Bombyx mori. Entomol Res. 35, 199205. 